\title{
Distribuição altitudinal e simpatria das aves do gênero Drymophila Swainson (Passeriformes, Thamnophilidae) na Mata Atlântica
}

\author{
Henrique Rajão \& Rui Cerqueira \\ Laboratório de Vertebrados, Departamento de Ecologia, Instituto de Biologia, Universidade Federal do Rio de Janeiro. Caixa \\ Postal 68020, 21941-590 Rio de Janeiro, Rio de Janeiro, Brasil. E-mail: rajao@biologia.ufrj.br
}

\begin{abstract}
Elevational distribution and sympatry of birds of the genus Drymophila Swainson (Passeriformes, Thamnophilidae) in the Atlantic forest. The elevational distribution of Atlantic forest Drymophila Swainson, 1824 was analyzed and sympatry cases were identified and discussed. Two data sets were used, one with local scale data (a portion of Serra do Mar called Serra dos Órgãos, in Rio de Janeiro state) and other with regional scale data (Atlantic forest). The Serra dos Órgãos records and elevations were obtained in the field while Atlantic forest data were compiled mainly following a survey of specimens deposited in natural history museums. Both data sets showed a similar pattern of elevational distribution: D. squamata (Lichtenstein, 1823) and D. ferruginea (Temminck, 1822) at lower elevations, D. malura (Temminck, 1825), D. ochropyga (Hellmayr, 1906) and D. rubricollis (Bertoni, 1901) all with similar, intermediate, elevational limits and D. genei (Filippi, 1847), at higher elevations. Most localities and observation stations presented only one or two species, suggesting that species tend to exclude each other spatially. In comparison, D. squamata and D. malura were the species with the lowest degree of mutual sympatry, probably reflecting distinct habitat preferences in relation to the other Drymophila species. Sympatry between sister species $D$. ochropyga-D. genei and D. ferruginea-D. rubricollis was observed at Serra do Mar and Serra da Mantiqueira localities, along narrow elevational bands, never wider than $300 \mathrm{~m}$.

KEY WORDS. Bamboo; biogeography; endemic species; Serra do Mar; Serra da Mantiqueira.
\end{abstract}

RESUMO. Foram descritas e analisadas as distribuições altitudinais das seis espécies de Drymophila Swainson, 1824, endêmicas da Mata Atlântica e identificados e discutidos os casos de simpatria. Para isso, foram usados dois conjuntos de dados, um em escala local (a Serra dos Órgãos, um segmento da Serra do Mar no Estado do Rio de Janeiro) e outro em escala regional (Mata Atlântica). Os registros e as altitudes na Serra dos Órgãos foram obtidos diretamente no campo enquanto que os dados da Mata Atlântica foram obtidos principalmente a partir de exemplares depositados em museus de história natural. Os dois conjuntos de dados mostraram um padrão similar de distribuição altitudinal das espécies: D. squamata (Lichtenstein, 1823) e D. ferruginea (Temminck, 1822) nas menores altitudes, D. malura (Temminck, 1825), D. ochropyga (Hellmayr, 1906) e D. rubricollis (Bertoni, 1901) com limites altitudinais semelhantes entre si e intermediários em relação às demais espécies e D. genei (Filippi, 1847), restrita às maiores altitudes. A maior parte das localidades na Mata Atlântica e pontos de observação na Serra dos Órgãos apresenta apenas uma ou duas espécies. Isso sugere que, embora possam ocorrer nas mesmas áreas ou localidades, as espécies tendem a se excluir. Drymophila squamata e $D$. malura foram as espécies que menos vezes ocorreram em simpatria proporcionalmente, nas duas escalas de análise. A simpatria entre as espécies irmãs $D$. ochropyga-D. genei e D. ferruginea-D. rubricollis ocorreu em localidades nas Serras do Mar e da Mantiqueira, sempre em faixas altitudinais estreitas, nunca superiores a $300 \mathrm{~m}$ de intervalo.

PALAVRAS CHAVE. Bambu; biogeografia; espécies endêmicas; Serra do Mar; Serra da Mantiqueira.

Drymophila Swainson, 1824, inclui pequenas aves insetívoras $(\mathrm{ca} .13 \mathrm{~cm})$, encontradas geralmente no sub-bosque de florestas primárias ou secundárias onde estão freqüentemente associadas a microhabitats específicos como taquarais, emaranhados de cipó e clareiras (Ridgely \& Tudor 1994, Parker et al. 1996, SicK 1997, Zimmer \& IsLER 2003). A maior parte das espécies mostra clara preferência por ambientes com bambu (Poaceae: Bambusoideae) (Parker 1982, Ridgely \& Tudor 1994, Parker et al. 1996, SicK 1997, Leme 2001a, b, Zimmer \& IsLer 2003).

Oito espécies compõem esse gênero, sendo seis delas endêmicas da Mata Atlântica (D. squamata (Lichtenstein, 1823), D. ferruginea (Temminck, 1822), D. rubricollis (Bertoni, 1901), 
D. genei (Filippi, 1847), D. ochropyga (Hellmayr, 1906) e D. malura (Temminck, 1825)), uma dos Andes (D. caudata (Sclater, 1855)) e uma amazônica (D. devillei (Menegaux \& Hellmayr, 1906)) (Ridgely \& Tudor 1994, Zimmer \& Isler 2003). O relacionamento filogenético entre as espécies do gênero foi recentemente estudado por J. Goerck, que conseguiu estabelecer, a partir de análises baseadas em seqüências de ADN mitocondrial, a existência de três clados bem resolvidos, formados pelos seguintes pares de espécies: $D$. ferruginea e $D$. rubricollis; $D$. genei e $D$. ochropyga; D. caudata e D. devillei (Zimmer \& IsLer 2003).

A distribuição geográfica do gênero Drymophila na Mata Atlântica se estende desde o Estado de Alagoas no norte até o Rio Grande do Sul, Paraguai e Argentina no sul. Drymophila ocorre do nível do mar até as regiões mais altas de algumas montanhas no sudeste brasileiro. As espécies se sobrepõem geograficamente e pode haver simpatria entre duas ou mais espécies (Ridgely \& Tudor 1994, Sick 1997, Zimmer \& Isler 2003).

Aspectos da especialização e segregação ecológica dessas espécies foram estudados por Leme (2001a, b) e por J. Goerck (dados não publicados) na tentativa de entender os mecanismos de manutenção da simpatria e sintopia. Nenhum estudo foi publicado até o momento, no entanto, analisando os padrões de distribuição altitudinal e de simpatria ao longo da Mata Atlântica. Para Leme (2001a), estudos biogeográficos e históricos podem ajudar a identificar os mecanismos que permitem a coexistência simpátrica e sintópica das espécies de Drymophila na Mata Atlântica.

Neste trabalho são analisados os fatores que permitem a coexistência entre as espécies de Drymophila na Mata Atlântica, procurando-se responder as seguintes questões: (1) quais são os limites altitudinais de cada espécie e como eles variam ao longo das distribuições geográficas?; (2) quais são as faixas altitudinais com maior riqueza de espécies?; (3) qual a extensão da sobreposição altitudinal entre as espécies, particularmente entre as espécies irmãs?; as espécies se excluem altitudinalmente?; (4) em que grau e onde as espécies estão ocorrendo em simpatria, particularmente as espécies irmãs?

\section{MATERIAL E MÉTODOS}

Foram usados dois conjuntos de dados, um em escala local (região da Serra dos Órgãos) e outro em escala regional (Mata Atlântica). A Serra dos Órgãos corresponde a um trecho da Serra do Mar localizado na região central do Estado do Rio de Janeiro. Os dados foram analisados separadamente e em conjunto.

Para o estudo de variação altitudinal na Serra dos Órgãos foram feitos registros de campo, tanto visuais quanto sonoros, em 25 pontos de observação, entre os anos de 1990 e 2004. Os pontos foram visitados geralmente na parte da manhã, entre 06:00 e 09:00 h, em datas aleatórias. Somente um dos pontos, o Parque Nacional da Serra dos Órgãos - Sede Teresópolis, foi visitado regularmente. As observações nesse ponto foram feitas a cada três meses, entre os anos de 2003 e 2004 . O número de dias de observação por ponto variou de um a 50 dias (Tab. I).
Os pontos de observação são fragmentos de mata primária ou secundária, de diversos tamanhos, localizados em diferentes altitudes nos municípios de Guapimirim e Teresópolis, na região serrana do Estado do Rio de Janeiro. Os nomes usados para os pontos, neste trabalho, geralmente correspondem a bairros ou distritos desses municípios e podem ser facilmente encontrados em cartas topográficas (Tab. I). Os pontos não são localidades no sentido que esse termo é usado nas análises em escala regional (nessa escala, por exemplo, Serra dos Órgãos representa uma única localidade, com um valor único de altitude associado). Os pontos mais próximos distaram entre si aproximadamente $5 \mathrm{~km}$ em linha reta enquanto os mais distantes distaram em torno de $50 \mathrm{~km}$. Esses pontos, em conjunto, cobrem todo o gradiente altitudinal da região, que vai desde o nível do mar até $2263 \mathrm{~m}$ de altitude, na Pedra do Sino, pico mais alto da Serra dos Órgãos.

As espécies foram identificadas no campo através de observação direta com binóculos ou através da vocalização. Gravações eventuais foram feitas usando gravador Sony TCM-5000 EV com microfone direcional Senheiser ME-66. As altitudes foram obtidas diretamente no campo com o uso de altímetro analógico Thommen, com intervalo de $10 \mathrm{~m}$. Os registros feitos em pontos de observação nos municípios de Guapimirim e Teresópolis, fora do Parque Nacional da Serra dos Órgãos foram cedidos por R. Parrini.

As coordenadas e altitudes das localidades usadas para as análises na Mata Atlântica foram, em grande parte, cedidas por P.H.C. Cordeiro. Os pontos de ocorrência para cada uma das espécies foram obtidos por Cordeiro nas etiquetas dos espécimes depositados em museus de história natural, na literatura ou através de registros de campo (para mais detalhes sobre a metodologia usada para a obtenção dos dados ver CoRDEIRo 2001). Adicionalmente foi feita uma revisão da literatura em busca de novos registros e de dados precisos e inequívocos de altitude. Foram compiladas 198 localidades para as seis espécies endêmicas da Mata Atlântica. Para as análises de simpatria na Mata Atlântica foram utilizadas todas as localidades compiladas.

Os dados de altitude em escala local diferem daqueles em escala regional, obtidos principalmente a partir de localidades assinaladas em exemplares de museu, pela precisão. Enquanto os primeiros são obtidos diretamente no campo, com o uso de altímetro, os últimos são obtidos por interpolação ou pela escolha de uma altitude representativa da localidade, desde que não haja indicação da altitude nas etiquetas das peles depositadas em museu ou nos registros da literatura. Assim, por exemplo, localidades como Teresópolis, que apresentam uma amplitude altitudinal de mais de mil metros, terão que ser representadas nas análises por um único valor de altitude a ser escolhido, seja por interpolação, seja pela escolha de uma altitude representativa ou, mais freqüentemente, pelo uso dos valores citados em listas geonímicas (CERQUeIra 1995). A altitude escolhida para representar uma localidade pode então não ser condizente com os limites altitudinais de uma dada espécie. 
Tabela I. Pontos de observação na região da Serra dos Órgãos, municípios de Guapimirim e Teresópolis, Rio de Janeiro, apresentados em ordem crescente de altitude.

\begin{tabular}{|c|c|c|c|c|}
\hline Ponto de observação & Latitude & Longitude & $\begin{array}{c}\text { Altitude } \\
\text { amostrada (m) }\end{array}$ & $\begin{array}{l}\text { Períodos de observação (número } \\
\text { de dias de observação) }\end{array}$ \\
\hline Guapimirim & $22^{\circ} 32^{\prime} \mathrm{S}$ & $42^{\circ} 59^{\prime} \mathrm{W}$ & $0-200$ & $1993-96(29)$ \\
\hline Monte Olivete & $22^{\circ} 31^{\prime} \mathrm{S}$ & $43^{\circ} 01^{\prime} \mathrm{W}$ & $200-400$ & $1993-95(8)$ \\
\hline Parque Nacional da Serra dos Órgãos - Sede Guapimirim & $22^{\circ} 29^{\prime} \mathrm{S}$ & $43^{\circ} 00^{\prime} \mathrm{W}$ & 500 & $1990,1992-93,1995-97(6)$ \\
\hline Garrafão & $22^{\circ} 28^{\prime} \mathrm{S}$ & $43^{\circ} 00^{\prime} \mathrm{W}$ & $500-700$ & $1992-97,1999-00(29)$ \\
\hline Vieira & $22^{\circ} 15^{\prime} \mathrm{S}$ & $42^{\circ} 46^{\prime} \mathrm{W}$ & 800 & $1991,1995(4)$ \\
\hline Carangola & $22^{\circ} 15^{\prime} \mathrm{S}$ & $42^{\circ} 46^{\prime} \mathrm{W}$ & $800-900$ & $1990(1)$ \\
\hline Água Quente (Volta do Pião) & $22^{\circ} 08^{\prime} \mathrm{S}$ & $42^{\circ} 45^{\prime} \mathrm{W}$ & $800-900$ & $1990,1992,1994,1996(4)$ \\
\hline Boa Fé & $22^{\circ} 22^{\prime} \mathrm{S}$ & $42^{\circ} 45^{\prime} \mathrm{W}$ & $800-900$ & $1990-97(22)$ \\
\hline Canoas & $22^{\circ} 24^{\prime} \mathrm{S}$ & $42^{\circ} 53^{\prime} w$ & $800-900$ & 1990-92, 1994-96, 1998 (26) \\
\hline Bom Sucesso & $22^{\circ} 15^{\prime} \mathrm{S}$ & $42^{\circ} 47^{\prime} \mathrm{W}$ & $800-950$ & $1991-92(3)$ \\
\hline Serra do Capim & $22^{\circ} 07^{\prime} \mathrm{S}$ & $42^{\circ} 46^{\prime} \mathrm{W}$ & $800-1000$ & $1990-96(12)$ \\
\hline Andradas & $22^{\circ} 04^{\prime} \mathrm{S}$ & $46^{\circ} 34^{\prime} \mathrm{W}$ & 850 & 1993, 1995 (3) \\
\hline Vargem Grande & $22^{\circ} 23^{\prime} \mathrm{S}$ & $42^{\circ} 52^{\prime} \mathrm{W}$ & 850 & $1991-92,1994-97(21)$ \\
\hline Querência do Imperador & $22^{\circ} 24^{\prime} \mathrm{S}$ & $42^{\circ} 53^{\prime} \mathrm{W}$ & 850 & $1990-97(22)$ \\
\hline Frades & $22^{\circ} 20^{\prime} \mathrm{S}$ & $42^{\circ} 46^{\prime} \mathrm{W}$ & 880 & 1990-94, $1996(11)$ \\
\hline Ponte Nova & $22^{\circ} 16^{\prime S}$ & $42^{\circ} 54^{\prime} \mathrm{W}$ & 900 & $1992(2)$ \\
\hline Serrinha (Tapera) & $22^{\circ} 18^{\prime} \mathrm{S}$ & $42^{\circ} 51^{\prime} \mathrm{W}$ & 900 & 1993-95 (3) \\
\hline Santo Amaro & $22^{\circ} 21^{\prime} \mathrm{S}$ & $42^{\circ} 53^{\prime} \mathrm{W}$ & $900-1000$ & $1993-95(3)$ \\
\hline Parque Nacional da Serra dos Órgãos - Sede Teresópolis & $22^{\circ} 26^{\prime} \mathrm{S}$ & $43^{\circ} 00^{\prime} \mathrm{W}$ & $900-2263$ & $1990-96,1999,2003-04(50)$ \\
\hline Campanha & $22^{\circ} 19^{\prime} \mathrm{S}$ & $42^{\circ} 49^{\prime} \mathrm{W}$ & 1000 & $1990,1996(2)$ \\
\hline Nhuguaçu & $22^{\circ} 30^{\prime} \mathrm{S}$ & $42^{\circ} 51^{\prime} \mathrm{W}$ & 1000 & 1990, $1994(2)$ \\
\hline Jacarandá & $22^{\circ} 25^{\prime} \mathrm{S}$ & $42^{\circ} 55^{\prime} \mathrm{W}$ & $1000-1100$ & $1991,1995-97,2000(10)$ \\
\hline Faz. Vale da Revolta & $22^{\circ} 26^{\prime} \mathrm{S}$ & $42^{\circ} 57^{\prime} \mathrm{W}$ & 1100 & $1991,1995-97,2000(10)$ \\
\hline Alto da Posse & $22^{\circ} 22^{\prime} \mathrm{S}$ & $42^{\circ} 51^{\prime} \mathrm{W}$ & 1200 & 1990-91, 1994-98, 2000 (13) \\
\hline Estrada Teresópolis-Petrópolis & $22^{\circ} 24^{\prime} \mathrm{S}$ & $43^{\circ} 00^{\prime} \mathrm{W}$ & 1450 & $1990,1994-96(10)$ \\
\hline
\end{tabular}

Por essa razão, a consistência dos dados da Mata Atlântica foi analisada e só foram considerados os dados de altitude condizentes com os limites altitudinais conhecidos das espécies na Mata Atlântica. As seguintes referências foram usadas para auxiliar na determinação das altitudes máxima e mínima das espécies: Scott \& BRoOKE 1985, Ridgely \& Tudor 1994, GonZaga et al. 1995, Lowen et al. 1997, Belton 2000, Vasconcelos \& Melo JÚNIOR 2001, ZIMMER \& ISLER 2003.

Para a determinação do gradiente altitudinal de riqueza de espécies foi conferido o número de espécies presentes a cada $100 \mathrm{~m}$ de altitude, assumindo-se que cada espécie ocorre continuamente entre os limites inferior e superior de suas distribuições altitudinais, ao menos nas escalas analisadas.

\section{Serra dos Órgãos}

\section{RESULTADOS}

Na Serra dos Órgãos, onde podem ser encontradas as seis espécies de Drymophila endêmicas da Mata Atlântica, foram ob- servadas as seguintes amplitudes altitudinais, sendo n o número de observações: $D$. squamata $-50-600 \mathrm{~m}(\mathrm{n}=18)$; $D$. ferruginea 300-1100 m (n =62); D. rubricollis - 900-1570 m (n = 44), D. genei $-1525-2125 \mathrm{~m}(\mathrm{n}=151) ;$ D. ochropyga $-800-1570 \mathrm{~m}(\mathrm{n}=57)$; D. malura - 800-1450 m ( $\mathrm{n}=48)$ (Fig. 1).

Drymophila genei foi a espécie que apresentou a menor sobreposição altitudinal com o conjunto das demais espécies (entre 1525 e $1570 \mathrm{~m}$ ), sendo que a sobreposição só ocorreu com $D$. rubricollis e $D$. ochropyga. As espécies que apresentaram maior sobreposição foram $D$. ochropyga e $D$. rubricollis (entre 900 e 1570 m) e D. ochropyga e D. malura (entre 800 e 1450 m). Essas três últimas espécies apresentaram uma sobreposição quase total de suas distribuições altitudinais. Drymophila squamata só se sobrepõe altitudinalmente com $D$. ferruginea, o que ocorre entre 300 e $600 \mathrm{~m}$. Essas duas espécies são as únicas que ocorrem ao nível do mar ou próximo a ele, nas baixadas e no sopé da Serra dos Órgãos. As demais espécies ou estão restritas a altitudes intermediárias $(D$. rubricollis, $D$. ochropyga e $D$.

Revista Brasileira de Zoologia 23 (3): 597-607, setembro, 2006 
malura) ou ocupam as cotas mais altas das montanhas da região, podendo mesmo alcançar a região dos campos de altitude, o que é o caso de $D$. genei (Fig. 1).

A sobreposição altitudinal entre as espécies irmãs $D$. ochropyga e D. genei ocorreu entre 1525 e 1570 m (Fig. 1). Essa faixa de sobreposição corresponde a 5,8 \% do gradiente altitudinal de $D$. ochropyga e a $7 \%$ do de $D$. genei. As duas espécies ocorreram juntas somente na Trilha da Pedra do Sino, no Parque Nacional da Serra dos Órgãos, onde foram observadas forrageando na mesma touceira de bambu uma única vez, a $1570 \mathrm{~m}$ de altitude.

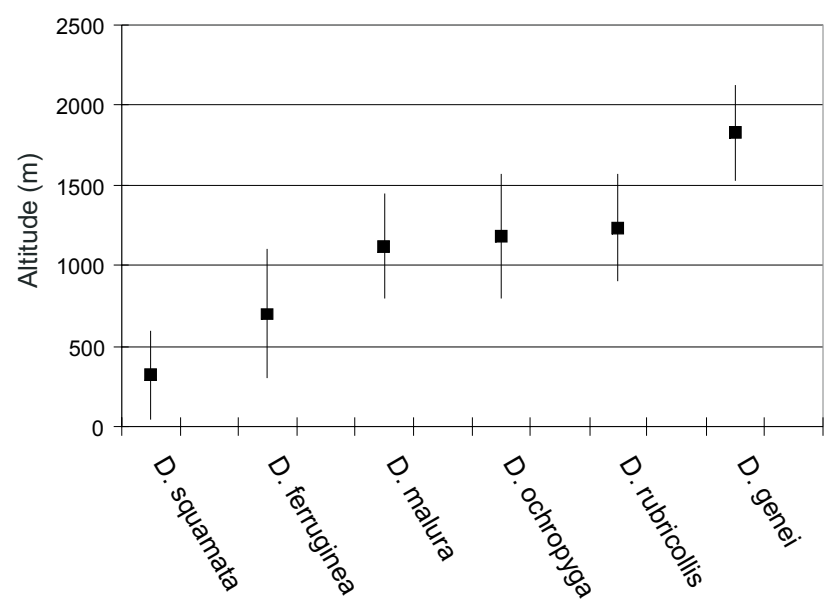

Figura 1. Amplitude de distribuição das altitudes das seis espécies de Drymophila encontradas na região da Serra dos Órgãos, Rio de Janeiro. As barras indicam os limites altitudinais máximo e mínimo. As espécies estão ordenadas, da esquerda para a direita, em ordem crescente dos pontos médios.

Em apenas dois pontos de observação (Canoas e Jacarandá) foi registrada a presença tanto de $D$. ferruginea quanto de D. rubricollis, sendo que a zona de sobreposição altitudinal entre essas duas espécies está situada entre 900 e $1100 \mathrm{~m}$, o que corresponde a $25 \%$ do intervalo altitudinal de ocorrência de $D$. ferruginea e a $29,8 \%$ do intervalo de D. rubricollis na Serra dos Órgãos.

A faixa de altitude com maior riqueza de espécies (quatro) está entre 900 e $1100 \mathrm{~m}$. As duas espécies que não ocorrem nessas altitudes são $D$. squamata e $D$. genei. Duas faixas altitudinais apresentaram uma única espécie: uma no sopé da Serra, abaixo de $300 \mathrm{~m}$, onde ocorre D.squamata e outra, acima de $1570 \mathrm{~m}$, onde nenhuma outra espécie foi encontrada a não ser D. genei (Fig. 2).

Em onze pontos de observação na Serra dos Órgãos (44\%) foi registrada apenas uma espécie. As espécies em que mais vezes ocorreram sozinhas proporcionalmente foram $D$. squamata ( $100 \%$ dos pontos) e D. malura ( $31 \%$ dos seus pontos de ocor- rência). Em oito pontos de observação (32\%) foram registradas duas espécies e em cinco pontos (20\%) três espécies. O maior número de espécies encontrado em um único ponto foi quatro (D. ferruginea, D. rubricollis, D. ochropyga e D. malura), o que ocorreu somente na localidade de Canoas $\left(22^{\circ} 24^{\prime} \mathrm{S}, 42^{\circ} 53^{\prime} \mathrm{W}\right)$, a $900 \mathrm{~m}$ de altitude.

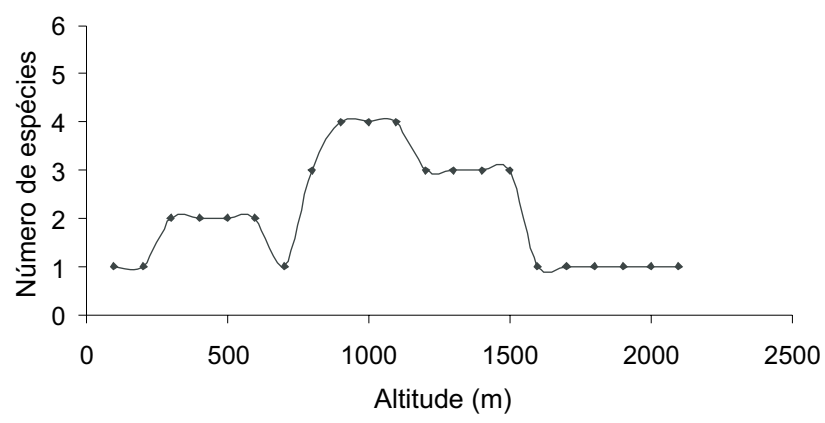

Figura 2. Distribuição da riqueza de espécies de Drymophila ao longo do gradiente altitudinal na região da Serra dos Órgãos, Rio de Janeiro.

\section{Mata Atlântica \\ Distribuição geográfica e simpatria}

O gênero Drymophila possui ampla distribuição na Mata Atlântica. As espécies que apresentam as maiores distribuições são D. ferruginea (Fig. 3) e D. squamata (Fig. 4), que ocorrem da Bahia à Santa Catarina. Drymophila squamata apresenta ainda uma população disjunta em Alagoas. Drymophila ochropyga (Fig. 5) ocorre do Espírito Santo à Santa Catarina, com populações disjuntas em áreas montanhosas da Bahia (Gonzaga et al. 1995, PARrini et al. 1999). A menor área de distribuição cabe a D. genei (Fig. 5), que é encontrada apenas nos Estados da região Sudeste. As duas espécies que se distribuem mais ao sul são $D$. rubricollis (Fig. 6) e $D$. malura (Fig. 7). Ambas ocorrem até o Rio Grande do Sul, extremo nordeste da Argentina (Misiones) e sudeste do Paraguai, sendo que, para o norte, D. rubricollis é encontrada até o Espírito Santo e D. malura até o leste de Minas Gerais e Sul da Bahia.

Não existem espécies com distribuições disjuntas. As distribuições geográficas das seis espécies de Drymophila endêmicas da Mata Atlântica se sobrepõem nos Estados do Rio de Janeiro, São Paulo e Minas Gerais, na região Sudeste do Brasil, entre as coordenadas $20^{\circ} 22^{\prime} \mathrm{S}$ e $22^{\circ} 45^{\prime} \mathrm{S}$ e $41^{\circ} 48^{\prime} \mathrm{W}$ e $45^{\circ} 35^{\prime} \mathrm{W}$, que correspondem aos limites de distribuição de D. genei.

Mais da metade das 198 localidades compiladas (56\%) possuem apenas uma espécie, sendo que as espécies que mais vezes ocorreram sozinhas proporcionalmente foram D. squamata (45\%) e D. malura (37\%). Em 54 localidades (27\%) foram registradas duas espécies, em 19 localidades (10\%) três espécies, em nove localidades (5\%) quatro espécies, em duas localidades (1\%) cinco espécies e em outras duas localidades (1\%) seis espécies. 

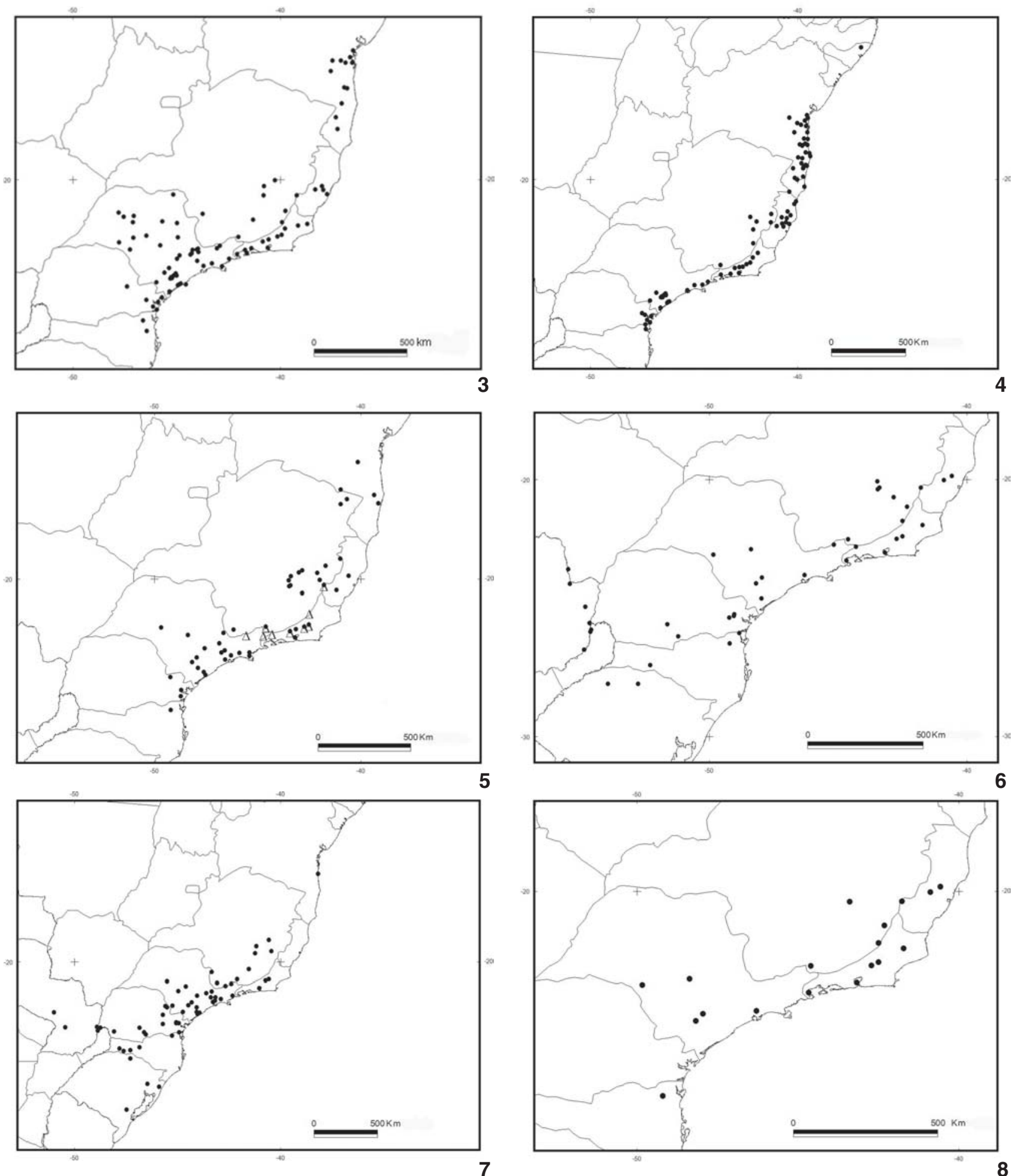

Figuras 3-8. Distribuição das aves do gênero Drymophila na Mata Atlântica: (3) Drymophila ferruginea; (4) Drymophila squamata; (5) Drymophila ochropyga $(\bullet)$ e Drymophila genei $(\Delta)$; (6) Drymophila rubricollis; (7) Drymophila malura; (8) Localidades onde ocorre simpatria entre $D$. ferruginea e $D$. rubricollis 
As duas localidades com maior riqueza de espécies, Serra dos Órgãos e Nova Friburgo, estão localizadas no Estado do Rio de Janeiro enquanto que, as duas localidades com cinco espécies, Rio de Janeiro (município) e Ribeirão Grande, estão localizadas respectivamente nos Estados do Rio de Janeiro e de São Paulo.

A simpatria entre as espécies irmãs $D$. ochropyga e $D$. genei foi observada em cinco localidades, entre as coordenadas $20^{\circ} 22^{\prime} \mathrm{S}$ e $22^{\circ} 36^{\prime} \mathrm{S}$ e $41^{\circ} 48^{\prime} \mathrm{W}$ e $44^{\circ} 38^{\prime} \mathrm{W}$, o que corresponde a $56 \%$ das localidades de $D$. genei e a $10 \%$ das localidades de $D$. ochropyga. Três dessas localidades estão situadas no Estado do Rio de Janeiro (Nova Friburgo, Serra do Tinguá e Serra dos Órgãos), uma na divisa entre Rio de Janeiro, São Paulo e Minas Gerais (Serra do Itatiaia) e uma entre os Estados do Espírito Santo e Minas Geras (Serra do Caparaó) (Fig. 5).

Drymophila rubricollis e D. ferruginea ocorrem em simpatria em 18 localidades, entre as coordenadas $19^{\circ} 55^{\prime} \mathrm{S}$ e $26^{\circ} 43^{\prime} \mathrm{S}$ e $40^{\circ} 36^{\prime} \mathrm{W}$ e $49^{\circ} 52^{\prime} \mathrm{W}$, o que corresponde a $20 \%$ das localidades de $D$. ferruginea e a quase metade das localidades de $D$. rubricollis (45\%). As localidades de simpatria pertencem aos Estados de Santa Catarina, São Paulo, Rio de Janeiro, Espírito Santo e Minas Gerais (Fig. 8).

\section{Limites altitudinais}

Uma vez que os limites altitudinais das espécies variaram ao longo das distribuições geográficas, principalmente com a latitude, optou-se por apresentá-los e definí-los caso a caso. Os gráficos das figuras 9 a 14 mostram a distribuição das localidades das seis espécies por altitude e latitude.

Drymophila squamata parece ocorrer principalmente até $600 \mathrm{~m}$ ao longo da maior parte de sua distribuição geográfica. Essa espécie aparentemente só alcança altitudes superiores a 800 m no Nordeste, mais especificamente no Estado da Bahia, onde também ocorre na baixada (Ridgely \& TUdOR 1994, GoNZAGA et al. 1995, PARKer et al. 1996, Zimmer \& Isler 2003). A maior parte das localidades de $D$. squamata está situada próximo ao nível do mar, até $200 \mathrm{~m}$, com alguns poucos pontos entre 200 e $600 \mathrm{~m}$ e com apenas dois pontos acima de $600 \mathrm{~m}$ (Itiruçu, a $820 \mathrm{~m}$ e Boa Nova, a 900 m, ambos no Estado da Bahia) (Fig. 9).

As localidades de $D$. ferruginea estão situadas principalmente até $1000 \mathrm{~m}$, com alguns poucos pontos acima dessa altitude (Fig. 10). Os pontos mais altos correspondem à Serra do Caraça, na porção meridional da Cadeia do Espinhaço, em Minas Gerais, onde D. ferruginea alcança $1300 \mathrm{~m}$ (VAsConcelos \& Melo Júnior 2001) e à Serra do Itatiaia, onde essa espécie ocorre até 1600 m (RIDGELY \& TUDOR 1994), embora seja visivelmente mais comum em menores altitudes, até ca. $1300 \mathrm{~m}$.

Drymophila rubricollis pode ser encontrada desde quase o nível do mar até $1950 \mathrm{~m}$. No entanto, não há evidências de que a distribuição altitudinal seja contínua. Essa espécie aparentemente só ocorre em baixas altitudes na parte sul de sua distribuição (Ridgely \& Tudor 1994, Zimmer \& Isler 2003), mais precisamente no extremo sudoeste, onde pode ocorrer em simpatria com $D$. malura. Registros feitos no Rio Grande do Sul e em áreas vizinhas no Paraguai e Argentina, confirmam a presença de D. rubricollis entre 100 e $500 \mathrm{~m}$ aproximadamente (Lowen et al. 1997, BeLton 2000). No restante da distribuição as altitudes parecem variar principalmente entre 750 e 1950 m (WILlis 1988, Ridgely \& TUdoR 1994, Vasconcelos \& Melo Júnior 2001, Zimmer \& Isler 2003). O gráfico da figura 11 mostra a concentração de pontos em baixas altitudes nas latitudes mais altas. Pontos mais altos localizados nessas mesmas latitudes correspondem a localidades litorâneas.

Drymophila genei está restrita às partes mais altas das montanhas do sudeste brasileiro (Fig. 12) e possui limites aparentemente bem definidos em sua pequena área de distribuição (ScotT \& Brooke 1985, Ridgely \& Tudor 1994, PARKer et al. 1996, Zimmer \& IsLER 2003). A partir dessas referências e de observações pessoais de H. Rajão, foi estabelecido que D. genei deve se distribuir principalmente entre 1150 e $2200 \mathrm{~m}$. Em pelo menos uma das localidades (Serra do Itatiaia), essa espécie ocorre continuamente ao longo de todo o intervalo altitudinal. De acordo com ZIMMER $\&$ Isler (2003), sem citar localidades ou detalhes de registros, $D$. genei pode ser encontrada ocasionalmente até $800 \mathrm{~m}$.

A maior parte das localidades de D. ochropyga está situada entre 500 e 1000 m (Fig. 13). Apesar de haver discordâncias na literatura em relação aos limites altitudinais dessa espécie, existem evidências da ocorrência de D. ochropyga entre 350 e 2000 m (BuzzetTi 2000, Vasconcelos \& Melo Júnior 2001), o que se aproxima muito dos limites considerados por ZiMMER \& IsLER (2003).

Drymophila malura ocorre desde as baixadas até $1900 \mathrm{~m}$ (e.g. Ridgely \& Tudor 1994, Parker et al. 1996, Belton 2000, ANJos 2002, ZimMER \& IsLER 2003). As localidades onde D. malura alcança as menores altitudes parecem estar concentradas especialmente na parte sul da distribuição (Fig. 14), onde essa espécie é simpátrica com $D$. rubricollis. Estabeleceu-se nesse trabalho como limite mínimo a altitude de $100 \mathrm{~m}$, o que pode ser observado em localidades como Paraguari e Itapuá, no Paraguai. A maior parte das localidades no entanto está situada entre 500 e 1000 m. Em uma única localidade, a Serra do Caraça, no Estado de Minas Gerais, D. malura alcança 1900 m (VASCONCELOS \& MELo JÚNIOR 2001).

Para o conjunto de dados da Mata Atlântica portanto, foram definidas as seguintes amplitudes altitudinais, sendo $\mathrm{n}$ o número de localidades compiladas para cada espécie: $D$. squamata - 5-900 m $(\mathrm{n}=81)$, D. ferruginea - 5-1600 $\mathrm{m}(\mathrm{n}=86)$, D. rubricollis $-100-1950 \mathrm{~m}(\mathrm{n}=35)$, D. genei $-1150-2200 \mathrm{~m}(\mathrm{n}=$ 7), D. ochropyga - 350-2000 m ( $\mathrm{n}=49)$, D. malura $-100-1900 \mathrm{~m}$ ( $\mathrm{n}=61$ ) (Fig. 15).

Todas as espécies, com exceção de D. genei podem ocorrer próximo ao nível do mar ao menos em parte de suas distribuições. As únicas duas espécies que não podem se sobrepor altitudinalmente são $D$. squamata e $D$. genei. Os limites máximo e mínimo de $D$. malura, $D$. ochropyga e $D$. rubricollis são semelhantes. Essas três espécies ocorrem desde as baixadas (abaixo de $500 \mathrm{~m}$ ) até $c a .2000 \mathrm{~m}$, o que se aproxima muito dos limites altitudinais do gênero na Mata Atlântica. Drymophila genei apresenta as maiores altitudes máxima e mínima entre 

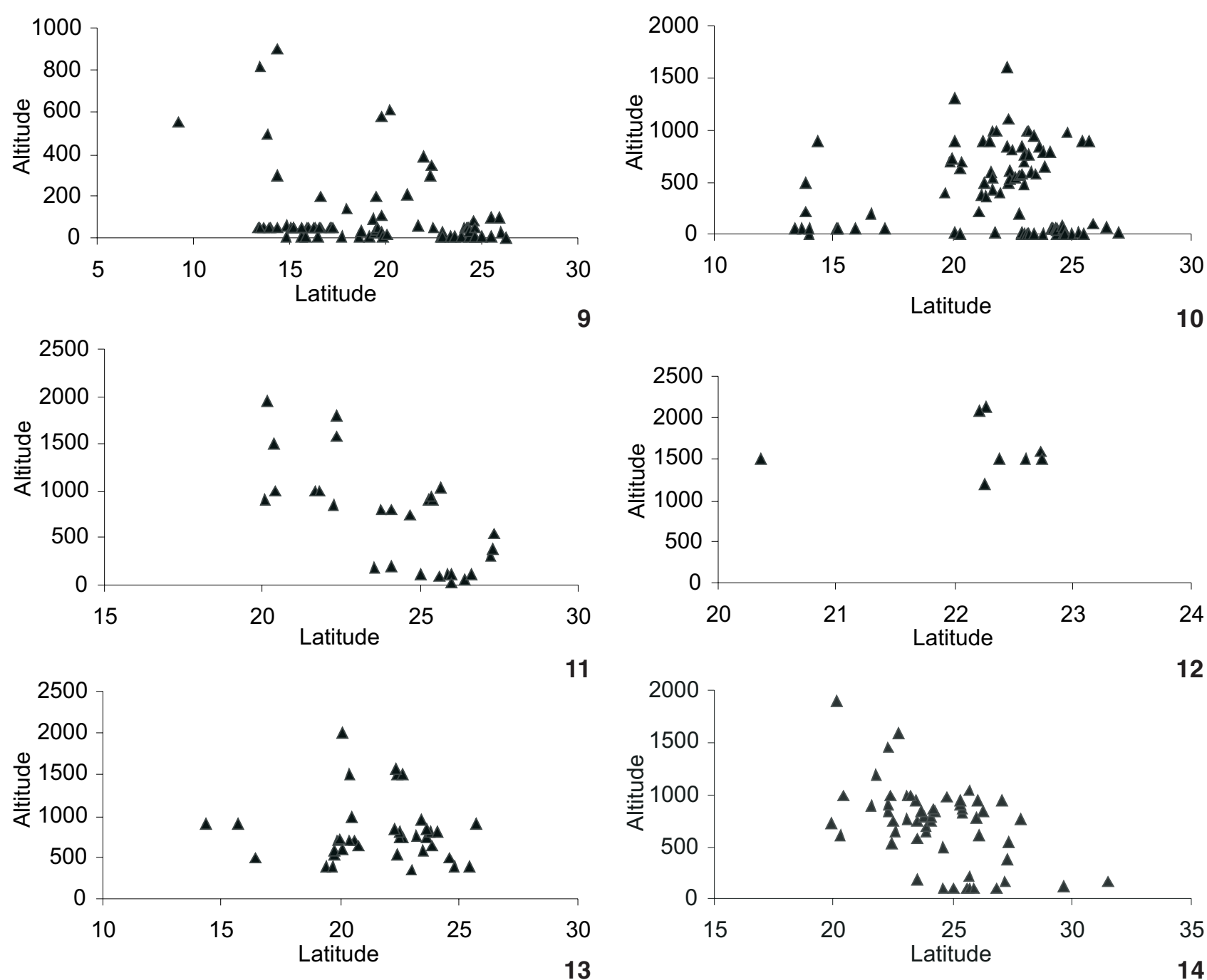

Figuras 9-14. Distribuição das localidades por altitude e latitude de: (9) D. squamata; (10) D. ferruginea; (11) D. rubricollis; (12) D. genei; (13) D. ochropyga; (14) D. malura.

todas as espécies enquanto D. squamata apresenta a menor mínima (junto com $D$. ferruginea) e a menor altitude máxima entre todas as espécies (Fig. 15).

Esses valores representam os limites altitudinais das seis espécies no conjunto da Mata Atlântica. Como foi visto que existem variações nesses limites ao longo da distribuição geográfica das espécies, os intervalos de sobreposição altitudinal mostrados na figura 15 são potenciais, podendo não se realizar nas áreas de simpatria.

A sobreposição altitudinal entre D. genei e D. ochropyga ocorre potencialmente entre 1150 e $2000 \mathrm{~m}$ (Fig. 15). Se forem consideradas, no entanto, somente as localidades onde foi observada simpatria entre essas duas espécies a sobreposição só poderá ocorrer entre 1150 e $1570 \mathrm{~m}$.
A sobreposição altitudinal entre $D$. ferruginea e $D$. rubricollis ocorre potencialmente entre 100 e 1600 m (Fig. 15). No entanto, se forem consideradas somente as localidades de simpatria, a sobreposição só poderá ocorrer entre 750 e 1600 m de altitude.

\section{Gradiente altitudinal de riqueza}

As distribuições altitudinais das espécies não são contínuas ao longo de suas distribuições geográficas. Sendo assim, para que fossem analisados os padrões de riqueza (número de espécies) por faixa altitudinal optou-se por considerar somente as localidades situadas dentro dos limites da área de sobreposição ente as seis espécies. Dentro desses limites não existem evidências de grandes descontinuidades nas altitudes, além do que, nessa área, não existem restrições geográficas, de latitude 


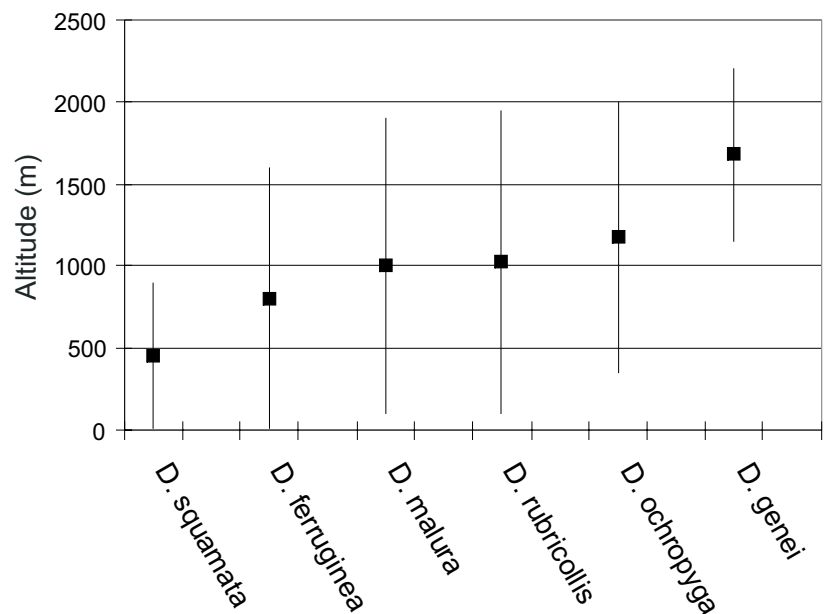

Figura 15. Amplitude de distribuição das altitudes das seis espécies de Drymophila encontradas na Mata Atlântica. As barras indicam os limites altitudinais máximo e mínimo. As espécies estão ordenadas, da esquerda para a direita, em ordem crescente dos pontos médios.

ou longitude, para a ocorrência do número máximo de espécies.

O gráfico da figura 16 mostra que há um crescimento contínuo no número de espécies com a altitude e um pico com cinco espécies entre 1200 e $1500 \mathrm{~m}$. Após essa faixa altitudinal ocorre uma diminuição contínua no número de espécies em direção às maiores altitudes.

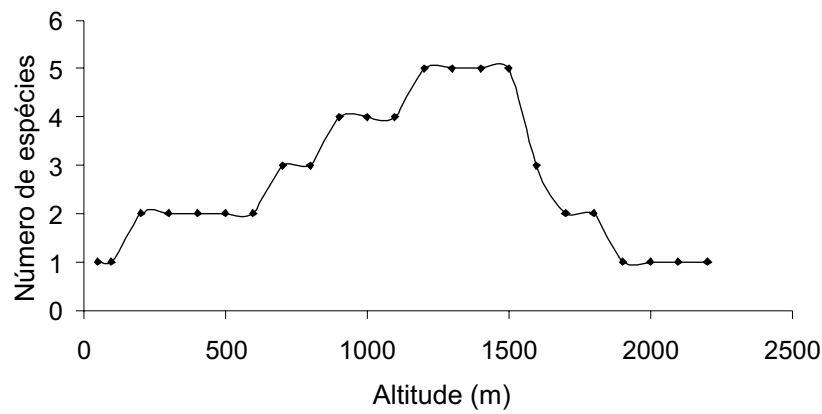

Figura 16. Distribuição da riqueza de espécies por altitude, consideradas apenas as localidades situadas na área de sobreposição entre as seis espécies de Drymophila na Mata Atlântica.

\section{DISCUSSÃO}

\section{Distribuição altitudinal}

A acurácia da determinação das altitudes é distinta nos dois conjuntos de dados aqui analisados. No entanto, há um padrão geral claramente similar nos dois conjuntos de dados. Tanto na Serra dos Órgãos quanto na Mata Atlântica como um todo, D. squamata e D. ferruginea ocupam as menores altitudes enquanto $D$. genei está restrita às partes mais altas das montanhas. Três espécies, $D$. malura, $D$. ochropyga e $D$. rubricollis possuem limites altitudinais semelhantes entre si e intermediários em relação às demais espécies.

Os dados de uma região em particular, a Serra dos Órgãos, mostram uma tendência a que a distribuição altitudinal das espécies se inicie em altitudes maiores. Assim, por exemplo, D. rubricollis, D. ochropyga e D. malura são encontradas apenas a partir de $800 \mathrm{~m}$ na Serra dos Órgãos, mas ocorrem desde quase o nível do mar no conjunto da Mata Atlântica. Dois aspectos devem ser levados em consideração ao serem analisadas essas diferenças: as características locais na Serra dos Órgãos e a variação dos limites altitudinais das espécies, principalmente com a latitude. Em relação ao primeiro caso, podese supor que as espécies talvez comecem a ocorrer a partir de cotas altitudinais mais altas na Serra dos Órgãos devido a alterações antrópicas na vegetação, assim como pelas baixas densidades de bambu nas cotas mais baixas. Sick (1997) menciona que espécies de aves da Floresta Atlântica Montana, encontradas geralmente acima de $500 \mathrm{~m}$ de altitude podem ocorrer em altitudes mais baixas ou mesmo ao nível do mar quando existem matas contínuas.

Por outro lado, diversas espécies endêmicas da Mata Atlântica, atualmente restritas às montanhas na parte norte de suas distribuições, ocorrem ao nível do mar ou próximo a ele nas baixadas costeiras mais ao sul (Gonzaga et al. 1995, Sick 1997). Esse parece ser o caso de $D$. ferruginea, comum desde o nível do mar até $100 \mathrm{~m}$ de altitude nas matas de baixada do litoral sul do Rio de Janeiro e norte de São Paulo (Goerck 1999, Buzzetтi 2000) e de D. ochropyga, registrada por Buzzetti (2000) a partir de $350 \mathrm{~m}$ no litoral sul do Rio de Janeiro. Drymophila malura e $D$. rubricollis também são encontradas próximo ao nível do mar no sul de suas distribuições, mas principalmente nas florestas interioranas da bacia do rio Paraná e não nas encostas e baixadas litorâneas. Esse mesmo fenômeno ocorre com bambus lenhosos (tribo Bambuseae) dos gêneros Guadua Kunth, 1822, Chusquea Kunth, 1822 e Merostachys Sprengel, 1824, comuns nas florestas atlânticas de baixa altitude da Argentina e Paraguai (Sтотz et al. 1996).

Os limites altitudinais máximos foram sempre superiores quando analisados os dados da Mata Atlântica como um todo. Drymophila squamata mostrou uma tendência a ocorrer em maiores altitudes nas latitudes mais baixas, o que ocorreu especificamente em localidades no Estado da Bahia. As demais espécies alcançaram as maiores altitudes sempre em serras interioranas como a Mantiqueira e o Espinhaço, corroborando as observações de WiLlis (1988) em relação a $D$. ferruginea e $D$. rubricollis. Drymophila genei e D. ferruginea ocorrem em altitudes superiores na Serra do Itatiaia, na Mantiqueira. Drymophila ochropyga, D. malura e D. rubricollis atingem seus limites máximos de altitude na Serra do Caraça, porção meridional da Cadeia do Espinhaço, em Minas Gerais. 
Todas as espécies têm suas distribuições correspondendo a das formações ombrófilas e semidecíduas atlânticas (sensu Oliveira-Filho \& Fontes 2000). A dificuldade em se determinar os padrões de distribuição altitudinal das espécies de Drymophila na Mata Atlântica provavelmente está relacionada com a mesma dificuldade encontrada por botânicos e fitogeógrafos em relação ao estabelecimento das faixas topográficas das diferentes formações florestais atlânticas (Veloso et al. 1991). A proposta de Veloso e colaboradores de que as faixas topográficas das diferentes formações ombrófilas (aluvial, terras baixas, submontana, montana e alto-montana) devam ser revistas e adaptadas de acordo com a escala do mapeamento deve ser considerada também no estudo da distribuição altitudinal das Drymophila e de outras aves florestais na Mata Atlântica.

\section{Gradiente altitudinal de riqueza}

A maior riqueza de espécies do gênero Drymophila na Mata Atlântica está nas altitudes médias, principalmente nas montanhas do sudeste brasileiro. Esse padrão foi encontrado também em outros estudos na região Neotropical, com comunidades de mamíferos (Geise et al. 2004) e de aves (Terborgh 1977, Sтотz et al. 1996). RAнвек (1995), a partir de extensa revisão de dados sobre a variação no número de espécies, tanto de animais como de plantas, com a altitude, identificou que, embora um declínio na riqueza de espécies com o aumento da altitude seja uma tendência geral, curvas em forma de corcunda ("humpshaped") parecem ser mais típicas do que aquelas que mostram um declínio monotônico. Terborgh (1977) argumentou que as altitudes médias, nos Andes, comportam mais espécies de aves porque apresentam maior heterogeneidade de ambientes, resultando em maior abundância de alimento, principalmente para as aves insetívoras.

Entre as seis espécies de Drymophila na Mata Atlântica, somente $D$. squamata (principalmente as populações meridionais) parece não apresentar qualquer preferência por bambu (Ridgely \& Tudor 1994, ZimMer \& Isler 2003). Formações de bam$\mathrm{bu}$, principalmente de bambus lenhosos dos gêneros Guadua, Chusquea e Merostachys, são provavelmente um recurso-chave para a ocorrência das Drymophila na Mata Atlântica (WiLLıs 1988, Ridgely \& Tudor 1994, Parker et al. 1996, Leme 2001a, Zimmer \& ISLER 2003)

A diversidade de bambus lenhosos é máxima na Região Neotropical no sudeste brasileiro entre as latitudes $20^{\circ}$ e $25^{\circ} \mathrm{S}$ (JuDzieWICZ et al. 1999). Bambus parecem ser mais abundantes, na Mata Atlântica, em maiores altitudes (WILLIs 1988), especialmente no sudeste brasileiro, onde as florestas montanas são normalmente dominadas por extensos bambuzais de Guadua, Chusquea e Merostachys (Sтотz et al. 1996). Extensas formações de bambus desses três gêneros estão presentes em altitudes médias, entre 800 e 1100 m no Parque Estadual Intervales, situado na porção da Serra do Mar conhecida como Serra de Paranapiacaba, no sul do Estado de São Paulo (Olmos 1991), mas não nas áreas mais baixas, até $350 \mathrm{~m}$ (Aleixo \& Galleti 1997). O mesmo fenômeno pode ser observado na Serra dos Órgãos e na Serra do Itatiaia.
A heterogeneidade de habitats nas montanhas do Sudeste brasileiro, em função do gradiente altitudinal, deve estar relacionada, de acordo com Cordeiro (2001) e Judziewicz et al. (1999), com a evolução ou ao menos com a manutenção de diversas espécies tanto de aves quanto de bambus. A heterogeneidade de habitats e a alta produtividade primária em altitudes médias, propostas por TERBoRgh (1977), podem não estar relacionadas com o processo de especiação das Drymophila nas montanhas da Mata Atlântica, mas antes proporcionando recursos suficientes para a coexistência dessas espécies.

\section{Simpatria}

Apesar da grande sobreposição entre as distribuições geográficas das espécies de Drymophila na Mata Atlântica, em mais da metade das localidades foi registrada apenas uma espécie (se forem consideradas as localidades com até duas espécies essa proporção aumenta para $83 \%$ ). As proporções na Serra dos Órgãos foram semelhantes, com $44 \%$ dos pontos de observação com apenas uma espécie e $76 \%$ com até duas espécies. Por outro lado, em apenas $2 \%$ das localidades, restritas às Serras do Mar e da Mantiqueira, no Sudeste brasileiro, foram registradas cinco ou seis espécies e em apenas um ponto de observação na Serra dos Órgãos foi registrado o número máximo de quatro espécies. Esses resultados sugerem que, embora possam ocorrer nas mesmas áreas ou localidades, as espécies tendem a se excluir independente da escala considerada.

Tanto em escala local quanto regional, $D$. squamata e $D$. malura foram as espécies que mais vezes ocorreram sozinhas proporcionalmente, apesar da grande sobreposição altitudinal com as demais espécies, principalmente em escala regional. Essas duas espécies são as que apresentam a menor associação com bambus na Mata Atlântica (Ridgely \& Tudor 1994, Leme 2001a, Zimmer \& Isler 2003), sendo consideradas como generalistas no uso de substratos de forrageio. A possibilidade de utilizar outros recursos que não bambu pode estar permitindo que essas espécies colonizem áreas na Mata Atlântica onde as outras espécies não podem ocorrer.

A distribuição geográfica de $D$. genei se sobrepõe inteiramente às áreas de distribuição de outras espécies do gênero, sendo que em todas as suas localidades ela ocorre em simpatria com outras espécies. Drymophila genei, no entanto, está entre as espécies com menor sobreposição altitudinal com as demais, tanto em escala local quanto regional.

A área de distribuição geográfica de D. ochropyga também se sobrepõe em grande parte às distribuições de outras espécies do gênero, assim como a sua faixa de ocorrência altitudinal. Além disso, D. ochropyga ocorre com outras espécies na maior parte das suas localidades e pontos de observação (73\% e $86 \%$ respectivamente). A especialização no uso de folhas mortas como substrato de forrageio, única no gênero, é provavelmente um dos fatores que permitem a ocorrência sintópica de $D$. ochropyga com as demais espécies de Drymophila (Leme 2001a, b, Zimmer \& IsLer 2003).

Drymophila ochropyga e D. genei ocorrem simpatricamente

Revista Brasileira de Zoologia 23 (3): 597-607, setembro, 2006 
nas Serras do Mar e da Mantiqueira, principalmente no Estado do Rio de Janeiro, onde estão situadas quatro das cinco localidades de simpatria. Em duas dessas localidades, Serra dos Órgãos e Serra do Itatiaia, representativas dos dois maciços, a faixa altitudinal de contato é bastante estreita (1525-1570 m e 1195$1350 \mathrm{~m}$ respectivamente), mostrando que talvez haja uma tendência à exclusão altitudinal entre essas duas espécies quando em simpatria. É possível que, na ausência de $D$. genei, $D$. ochropyga possa estender sua distribuição até maiores altitudes, como ocorre na Serra do Caraça, em Minas Gerais, onde essa espécie atinge seu limite altitudinal máximo, de $2000 \mathrm{~m}$. Estudos em outras áreas de simpatria poderão verificar se a tendência observada de segregação altitudinal entre essas espécies é generalizada.

Drymophila ferruginea e $D$. rubricollis ocorrem em simpatria ao longo das Serras do Mar e da Mantiqueira, sendo que $D$. ferruginea é espécie mais costeira e setentrional, enquanto $D$. rubricollis é mais meridional e se distribui mais para o interior (Fig. 17). Ao longo da área de simpatria entre $D$. ferruginea e $D$. rubricollis, assim como ocorre com D. genei e D. ochropyga, uma das espécies é exclusivamente montana ou alto-montana ( $D$. rubricollis) enquanto a outra ocorre tanto nas baixadas quanto nas montanhas, embora alcançando apenas altitudes médias ( $D$. ferruginea) (Fig. 18). A sobreposição altitudinal entre elas portanto está situada em altitudes médias, podendo ocorrer principalmente entre 750 e $1300 \mathrm{~m}$. Em diversas localidades de simpatria no entanto, de maneira similar ao ocorrido com $D$. genei e $D$. ochropyga, a faixa de sobreposição altitudinal mostrou ser bem mais estreita e nunca superior a $300 \mathrm{~m}$ de intervalo, como observado na Serra dos Órgãos (esse artigo), na Serra do Itatiaia (Ridgely \& Tudor 1994), em Parati (BuzzeTtr 2000) e em várias localidades no Estado de São Paulo (Willis 1988, Leme 2001a).

Estudos sobre comportamento de forrageio conduzidos em diversas localidades da Mata Atlântica (Leme 2001a, J. Goerck, dados não publicados) mostraram que $D$. ferruginea e $D$. rubricollis têm as mesmas preferências de microhabitat (ambas são especialistas em bambu) e que ambas forrageiam preferencialmente em folhas verdes. LEME (2001a) demonstrou ainda que ambas executam manobras de ataque à presa de forma semelhante concluindo que a sobreposição de nicho seria maior entre essas duas espécies do que entre qualquer outro par de espécies considerados (D. genei e D. squamata não entraram na análise de A. Leme).

A coexistência entre $D$. ferruginea e $D$. rubricollis, apesar da grande sobreposição ecológica, deve ser possível graças à abundância de recursos nas localidades de simpatria (LemE 2001a). A grande abundância e diversidade de bambus em altitudes médias nas serras do sudeste brasileiro, como já discutido anteriormente, deve estar permitindo que essas espécies ocorram lado a lado, ainda que em faixas altitudinais estreitas, sem que haja exclusão competitiva.

Concluindo, pode-se dizer que, a despeito da grande sobreposição entre as distribuições geográficas das espécies de Drymophila na Mata Atlântica, as espécies estão se excluindo localmente com, no máximo, duas espécies ocorrendo na gran- de maioria das localidades e altitudinalmente ainda que de maneira parcial na maioria dos casos. A coexistência entre várias espécies é possível em poucas localidades, situadas principalmente nas serras do Sudeste brasileiro, e em altitudes médias, onde a diversidade e a abundância de bambus parecem ser máximas e portanto a oferta de recursos não deve ser um fator limitante.
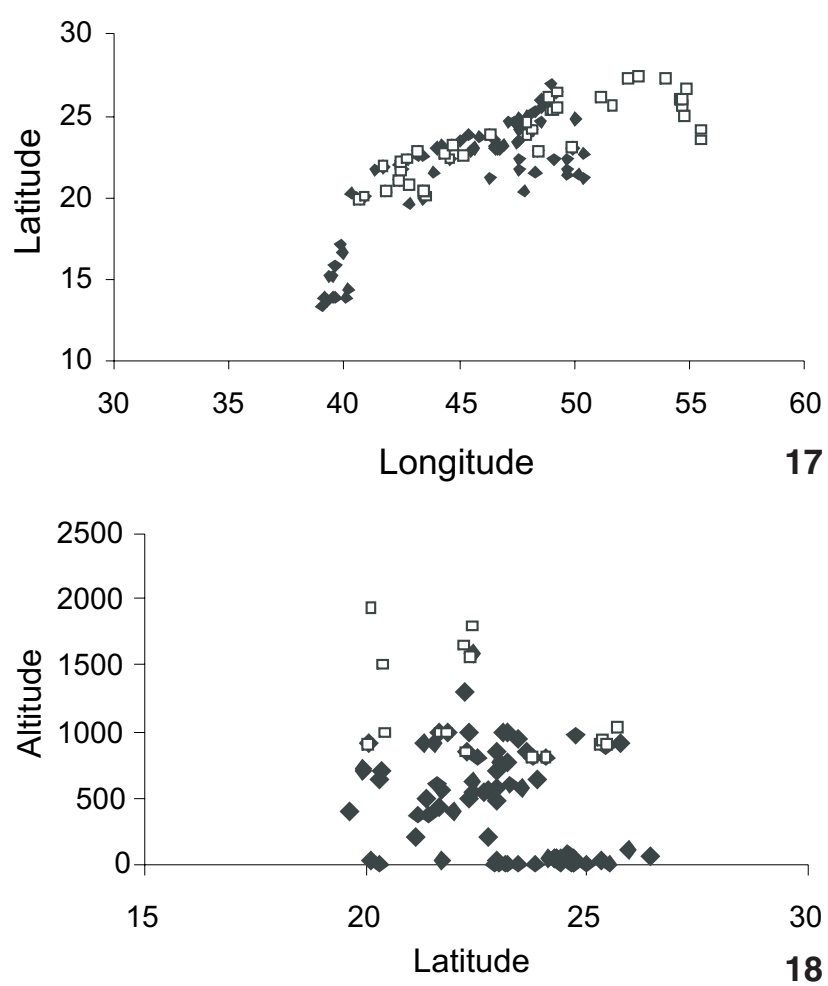

Figuras 17-18. Distribuição por: (17) longitude e latitude e (18) altitude e latitude, das espécies-irmãs $D$. ferruginea $(\diamond)$ e $D$. rubricollis $(\square)$ ao longo da área de simpatria.

\section{AGRADECIMENTOS}

A Ricardo Parrini, Paulo C. Cordeiro, Jaqueline Goerck, Luiz A.P. Gonzaga, José F. Pacheco, Carlos E. de V. Grelle e a dois consultores anônimos. À CAPES (bolsa de Henrique Rajão) e ao CNPq e FAPERJ (auxílios e bolsas a Rui Cerqueira).

\section{REFERÊNCIAS BIBLIOGRÁFICAS}

Aleixo, A. \& M. Galleti. 1997. The conservation of the avifauna in a lowland Atlantic forest in south-east Brazil. Bird Conservation International, Cambridge, 7: 235-261.

Anjos, L. Dos. 2002. Forest bird communities in the Tibagi river hydrographic basin, southern Brazil. Ecotropica, Ulm, 8: 67-79. 
Belton, W. 2000. Aves do Rio Grande do Sul: Distribuição e biologia. São Leopoldo, Ed. Unisinos, 584p.

BuzzetTI, D.R.C. 2000. Distribuição altitudinal de aves em Angra dos Reis e Parati, sul do Estado do Rio de Janeiro, Brasil, p. 131-148. In: M.A.S. Alves; J.M.C. Silva; M. Van Sluys; H.G. Bergallo \& C.F.D. Rocha. (Eds). A Ornitologia no Brasil: pesquisa atual e perspectivas. Rio de Janeiro, Editora da Universidade do Estado do Rio de Janeiro, 351p.

Cerqueira, R. 1995. Determinação de distribuições potenciais de espécies, p. 141-161 In: P. Peres-Neto; J.L. Valentin \& F.A.S. Fernandes (Eds). Oecologia Brasiliensis. Tópicos em Tratamento de Dados Biológicos. Rio de Janeiro, Programa de Pós-Graduação em Ecologia, Instituto de Biologia, Universidade Federal do Rio de Janeiro, vol. 2.

Cordeiro, P.H.C. 2001. Areografia dos Passeriformes endêmicos da Mata Atlântica. Ararajuba, Seropédica, 9 (2): 125-137.

Geise, L.; L.G. Pereira; D.E.P. Bossi \& H.G. Bergallo. 2004. Pattern of elevational distribution and richness of non volant mammals in Itatiaia National Park and its surroundings, in Southern Brazil. Brazilian Journal of Biology, Rio de Janeiro, 64 (3B): 599-612.

Goerck, J.M. 1999. Distribution of birds along an elevational gradient in the Atlantic forest of Brazil: implications for the conservation of endemic and endangered species. Bird Conservation International, Manchester, 9: 235-253.

Gonzaga, L.P.; J.F. Pacheco; C. Bauer \& G.D.A. Castiglioni. 1995. An avifaunal survey of the vanishing montane Atlantic forest of southern Bahia, Brazil. Bird Conservation International, Manchester, 5: 279-290.

Judziewicz, E.J.; L.G. Clark; X. Londoño \& M.J. STern. 1999. American bamboos. Washington, Smithsonian Institution Press, 392p.

Leme, A. 2001a. Foraging patterns and resource use in four sympatric species of antwrens. Journal of Field Ornithology, Tulsa, Oklahoma, 72 (2): 221-227.

Leme, A. 2001b. Foraging substrate selection by ochre-rumped antbird Drymophila ochropyga. Ararajuba, Seropédica, 9 (1): $7-11$.

Lowen, J.C.; R.P. Clay; J.M. Barnett; A. Madroño; M. Pearman; B.L. Lanús; J.A. Tobias; D.C. Liley; T.M. Brooks; E.Z. EsQuivel \& J.M. ReID. 1997. New and noteworthy observations on the Paraguayan avifauna. The Bulletin of the British Ornithologist's Club, Oxford, 117 (4): 275-293.

Oliveira-Filho, A. \& M.A.L. Fontes. 2000. Patterns of floristic differentiation among Atlantic forests in south-eastern
Brazil, and the influence of climate. Biotropica, Lawrence, 32: 793-810.

OLmos, F. 1991. Observations on the behavior and population dynamics of some Brazilian Atlantic Forest rodents. Mammalia, Paris, 55 (4): 555-565.

PARKER, T.A. 1982. Observations of some unusual rainforest and marsh birds in southeastern Peru. Wilson Bulletin, Fort Collins, 94 (4): 477-493.

Parker, T.A.; D.F. Stotz \& J.W. Fitzpatrick. 1996. Ecological and distributional databases, p. 131-146. In: D.F. Sтотz; J.W. FitzPatrick; T.A. Parker \& D.K. Moskovits (Eds). Neotropical birds: ecology and conservation. Chicago, University of Chicago Press, 478p.

Parrini, R.; M.A. Raposo; J.F. Pacheco; A.M.P. Carvalhaes; T.A. MeloJúnior; P.S.M. FonseCA \& J.C. Minns. 1999. Birds of the Chapada Diamantina, Bahia, Brazil. Cotinga, Bedfordshire, 11: 86-95.

RAнвEк, C. 1995. The elevational gradient of species richness: a uniform pattern? Ecography, Lund, 18: 200-205.

Ridgely, R. \& G. Tudor. 1994. The birds of South America. Austin, University of Texas Press, vol. 2, XII+814p.

SсотT, D. A. \& M. DE L. BRooke. 1985. The endangered avifauna of southeastern Brazil: a report on the BOU/WWF expeditions of 1980/81 and 1981/82. ICBP Technical Publication, Manchester, 4: 115-139.

SICK, H. 1997. Ornitologia Brasileira. Rio de Janeiro, Editora Nova Fronteira, 912p.

Stotz, D.F.; J. Fitzpatrick; T.A. Parker III \& D.K. Moskovits. 1996. Neotropical birds: ecology and conservation. Chicago, The University of Chicago Press, 478p.

Terborgh, J. 1977. Bird species diversity on an Andean elevational gradient. Ecology, Washington, 58: 1007-1019.

Vasconcelos, M.F. \& T.A. Melo Júnior. 2001. An ornithological survey of Serra do Caraça, Minas Gerais, Brazil. Cotinga, Bedfordshire, 15: 21-31.

Veloso, H.P.; A.L.R. Rangel Filho \& J.C.A. Lima. 1991. Classificação da vegetação brasileira, adaptada a um sistema universal. Rio de Janeiro, Instituto Brasileiro de Geografia e Estatísca, 123p.

Willis, E.O. 1988. Drymophila rubricollis (Bertoni, 1901) is a valid species (Aves, Formicariidae). Revista Brasileira de Biologia, Rio de Janeiro, 48 (3): 431-438.

Zimmer, K.J. \& M.L. IsLer. 2003. Family Thamnophilidae (Typical Antbirds), p. 448-681. In: J. Del Hoyo; A. Elliott \& D.A. CHRISTIE (Eds). Handbook of the Birds of the World. Barcelona, Lynx Edicions, vol. 8, 845p. 